Article original

\title{
Criblage de variétés commerciales de canne à sucre prometteuses dans le périmètre sucrier de ferké 2 au nord de la Côte d'Ivoire : optimisation de la durée de sélection
}

\author{
Didier K. KouAmÉ ${ }^{*}$, Crépin Bi PÉnÉ² \& Michel ZouZOu ${ }^{3}$ \\ ${ }^{1}$ Laboratoire de Physiologie Végétale, UFR Biosciences, Université de Cocody (Abidjan, Côte d'lvoire) et à SUCAF- Cl, site de \\ Ferké 2. 22 BP 582 Abidjan 22 (Côte d'lvoire), \\ 2Direction Recherche et Développement, SUCAF-Cl, 33 Rue des Brasseurs 01 BP 1967 Abidjan 01 (Côte d'Ivoire), \\ ${ }^{3}$ Laboratoire de Physiologie Végétale, UFR Biosciences, Université de Cocody, 22 BP 582 Abidjan 22 (Côte d'lvoire). \\ *Auteur pour les correspondances (E-mail : didykonan@yahoo.fr) \\ Reçu 08-10-2009, accepté le 06-05-2010
}

\begin{abstract}
Résumé
L'étude a été conduite en cinq ans à SUCAF/Ferké 2 au Nord de la Côte d'Ivoire dans le but de cribler des variétés précoces de canne à sucre sur la base du rendement en sucre et de la tolérance au charbon (U. scitaminea) et au foreur de tiges ( $E$. saccharina), principales contraintes biotiques du site. Les variétés SP70-1143 et SP71-1406 ont été sélectionnées ; elles ont présenté de très bons rendements agro-technologiques (12,2 et 15,31 t de sucre.ha1) et une tolérance au charbon (194,6 et 455,4 fouets/ha) et au foreur de tiges (1,21 et 0,94 entre-nœuds attaqués). SP71-1406 est une variété auto-épaillante à port érigé qui pourrait se prêter à la récolte mécanisée et à la culture en rangs jumelés. L'étude suggère en outre la pertinence d'importer des variétés commerciales et de mener la phase expérimentale de la sélection en une seule étape de trois ans.
\end{abstract}

Mot clés : sélection variétale, canne à sucre, variété, rendement, Côte d'Ivoire

\begin{abstract}
Screening of promising commercial sugarcane varieties at Ferké 2 sugar complex in northern ivory coast: optimization of the screening process duration.

Nine cane varieties were tested over five years at the early season on Ferké 2 sugar complex in northern Ivory Coast. Two commercial genotypes of Brazilian origin (SP70-1143 and SP71-1406) came out as high sugar yielding (12.2 and 15.3 t.ha $^{-1}$ ) and tolerant to the stalk borer as well as the smut (U. scitaminea) with 1.2 and 0.9 p.c of attacked stalk and 194.6 et 455.4 smutted stalk. ha ${ }^{-1}$. The later genotype with erected architecture and fewer straws is proned to a mechanized harvesting as well as dual row spacing. The study suggests the relevance of importing commercial cane varieties in Ivory Coast as well as carrying out the screening stage at three years.
\end{abstract}

Key words: screening, sugarcane, variety, yield, Ivory Coast.

\section{Introduction}

En Côte d'Ivoire, les premiers essais en vue de l'implantation de l'industrie sucrière ont débuté en 1960. Plusieurs points d'essais avaient été créés et six ont été retenus pour accueillir les complexes sucriers sous l'égide de l'exSODESUCRE. Aujourd'hui, quatre d'entre eux ont 
été maintenus en exploitation ; à savoir BorotouKoro, Ferké 1 et 2 au Nord et Zuénoula au CentreOuest du pays. Sur ces sites des variétés de canne telles que NCo376 et Co957 ont été introduites en 1960 et 1974 . Elles occupent des superficies importantes à Ferké 2, soit 15 et $30 \%$ en 20082009. Leurs rendements moyens en canne sont relativement faibles, soit 68 à 69 t.ha $^{-1}$, à raison de 77 t.ha $^{-1}$ sous irrigation et 42 t.ha $^{-1}$ en pluvial. C'est ce qui explique en partie le plafonnement de la production sucrière de la SUCAF-CI à 92000 t.an${ }^{1}$. Ces variétés cultivées aussi sur les autres complexes sucriers ivoiriens et d'Afrique de l'Ouest présentent par ailleurs une sensibilité marquée aux principales contraintes phytosanitaires de la canne à sucre en Côte d'Ivoire. Ces contraintes sont essentiellement le charbon dû à Ustilago scitaminea et le foreur de tiges Eldana saccharina, qui provoque des dégâts important en Afrique (Tran, 1981 ; Wada, 2003 ; Goebel et al., 2005 ; Goebel et al., 2008 ; Yoseph et al., 2008). Sur les sites de SUCAF/Ferké, le matériel végétal et l'eau apparaissent comme les premiers facteurs limitants de la productivité (Péné et al., 1997). Plus globalement, l'amélioration des pratiques culturales et une approche intégrée de celles-ci restent un important défi à relever afin de réduire les coûts de production. Ce qui aura pour conséquence d'accroître la compétitivité de la filière canne à sucre en Côte d'Ivoire. Cependant le schéma de sélection de la canne à sucre dure 15 ans et n'est pas favorable à la diffusion des variétés performantes sur le court terme. II est apparu donc nécessaire de faire face à la moindre productivité des complexes en criblant de nouvelles variétés performantes dans un schéma de sélection allégé qui permettrait à court terme de relever la production. Aussi, éviterait-on à l'industrie sucrière ivoirienne de rester en marge du progrès génétique en cours dans les autres pays où de nouvelles variétés améliorées ont déjà été déployées (Paulet \& Glaszmann, 1994; Ramdoyal \& Autrey, 2005 ; Domaingue et al., 2008). Ainsi, cette étude vise à contribuer de façon significative à l'amélioration de la productivité des complexes sucriers de la Côte d'Ivoire. II s'agit de cribler des variétés améliorées prometteuses à même de remplacer les variétés obsolètes existant sur les complexes depuis 1974. Une observation sur la possibilité de réduction de la phase expérimentale de sélection de la canne à sucre est faite dans cette étude.

\section{Matériel et méthodes}

\subsection{Site expérimental}

Le complexe sucrier de Ferké 2 est situé au Nord de la Côte d'Ivoire $\left(9^{\circ} 14^{\prime}-9^{\circ} 35^{\prime}\right.$ latitude Nord, 515'- 5०24' longitude Ouest, 323 m d'altitude). Son climat est de type tropical subhumide avec une saison sèche marquée par l'harmattan (vent sec et chaud) de novembre à mars et une saison pluvieuse d'avril à octobre. La pluviométrie moyenne annuelle est de $1200 \mathrm{~mm}$, les températures maximale et minimale sont de 32,5 et $21^{\circ} \mathrm{C}$. La durée de l'insolation avoisine 2610 h. $a n^{-1}$. Les sols du site sont hydromorphes, alluviaux (terrasses du fleuve Bandama) et ferrallitiques (prédominants), avec une couche arable peu profonde (40 à $60 \mathrm{~cm}$ ) due à la présence de carapaces (Eldin, 1971; John, 1975 ; Bigot et al., 2005).

\subsection{Matériel végétal}

Neuf variétés de canne, introduites en Côte d'Ivoire de 1983 à 1987 ont été criblées dans deux essais conduits chacun sur cinq ans. II s'agit, de quatre variétés commerciales (B47258, B51129 de Barbade et SP70-1143, SP71-1406 du Brésil) et de cinq variétés présélectionnées en Guadeloupe par le CIRAD (FR80674, FR80959, FR81254, FR81258 et FR8183) pour être évaluées dans les pays africains (Feldmann et al., 1998). L'essai 1 comprenait B47258, B51129, SP70-1143, FR80674 et FR80959 et l'essai 2, FR81254, FR81258, FR8183 et SP711406. Les variétés témoins sont NCo376 (de Natal et Coimbatore; bonnes qualités technologiques, sensible au charbon et au foreur) et Co449 (de Coimbatore ; résistante au charbon et au foreur). Toutes ces variétés sont des hybrides interspécifiques de Saccharum officinarum et S. spontaneum (Sreenivasan et al., 1987 ; D’Hont et al., 1996).

\subsection{Méthodes}

\subsubsection{Dispositif expérimental}

Chaque essai a été mis en place sur le site de Ferké 2, en début de saison de récolte (décembrejanvier) sur une période de cinq ans, selon un 
dispositif en blocs complets randomisés à un facteur étudié (variétés) répétées six fois. Chaque traitement comportait huit lignes de cannes d'une superficie de $102 \mathrm{~m}^{2}(8,5 \mathrm{~m} \times 1,5 \mathrm{~m} \times 8$ lignes $)$, dont $76,5 \mathrm{~m}^{2}$ correspondant aux six lignes récoltables; les deux lignes restantes servant de bordures.

\subsubsection{Analyses saccharimétriques}

Un échantillon de 30 cannes est prélevé dans chacune des parcelles élémentaires des essais et ramené au laboratoire. Chaque canne est coupée en trois morceaux, en conservant, soit une base, un milieu ou un bout pour constituer un échantillon secondaire de dix cannes reconstituées. Ce dernier est réduit en pulpe par un broyeur électrique (« Jeffco» food and fodder cutter grinder, model 265B taille 10, série L1710). Celle-ci est soumise à une presse hydraulique (pinette Emidecau Ind. 125). A partir de l'extrait recueilli, le Brix est mesuré à l'aide d'un réfractomètre (Schmidt+Haensch, model DURSW, série 29129) à $20^{\circ} \mathrm{C}$. Une partie du jus de canne est clarifiée selon la méthode de Horne par ajout d'acétate de plomb basique (hydroxideacétate de plomb (II) ou sel de Horne) à $2,5 \mathrm{~g}$ pour $250 \mathrm{ml}$ de jus non dilué (méthode ICUMSA GS5/7-1, 1994 citée par Hoareau et al., 2008). Après filtration du jus sur papier Whatman 91, le Pol est lu à l'aide d'un polarimètre (saccharomat Z, série 29305). La table de Schmidt relative au saccharimètre permet de déterminer le Pol du jus à partir du Brix et du Pol lu. La pureté du jus correspond au taux de Pol dans le Brix. Le taux de fibre est déterminé à l'aide d'une table de correspondance à partir du poids du gâteau (fibre) obtenu après pressage du broyat. La richesse saccharine est déterminée en multipliant le Pol du jus par un facteur $n$ lu sur une seconde table pour un poids de gâteau de $500 \mathrm{~g}$ de pulpe de canne (Hoarau, 1970). Enfin, le taux de sucre extractible (SE \%) est déterminé :

$S E \%=[(0,84 \times \mathrm{Pol} \%$ C) $(1,6-60 /$ Pureté $)-(0,05 \times$ Fibre \% C)]

Le rendement en sucre extractible (critère d'évaluation le plus pertinent) qui prend en compte le rendement en canne et les qualités technologiques est alors calculé comme suit :

$$
T S E . h a^{-1}=\frac{S E \% \times T C \cdot h a^{-1}}{100}
$$

\subsubsection{Suivi phytosanitaire}

La tolérance au charbon, principale maladie endémique due à $U$. scitaminea, et aux attaques du foreur de tiges ( $E$. saccharina) est un critère phytosanitaire pris en compte dans l'évaluation des variétés. Le comptage des fouets charbonneux sur chaque ligne utile commence à trois mois et demi après la plantation ou la coupe précédente (début de formation des tiges et des fouets). Le nombre de fouets. ha ${ }^{-1}$ est calculé comme suit:

Nombre de fouets. ha $^{-1}=$ nombre total fouets $\mathrm{x}$ 10000 / superficie utile ou récoltable

Le taux d'entre-nœuds attaqués est déterminé à la récolte. Un échantillon de 30 cannes est prélevé au hasard sur les six lignes récoltables à raison de cinq cannes par ligne. Ces cannes sont coupées longitudinalement et les entrenœuds sont observés en vue de déceler une éventuelle attaque du foreur (trou annulaire et coloration rougeâtre de la pulpe). Le nombre d'entre nœuds attaqués est rapporté au nombre total d'entre-nœuds sur chaque tige afin de calculer le taux d'attaque (ENA \%).

\subsubsection{Analyses statistiques}

Une analyse de variance à été appliquée aux paramètres mesurés et la comparaison des moyennes a été effectuée selon le test de Newman-Keuls (test post-hoc ANOVA) au seuil de signification de $5 \%$ à l'aide du logiciel STATISTICA 6.0. II s'en est suivi la caractérisation des groupes de variétés par l'analyse en composantes principales (ACP) à l'aide du même logiciel. Les cinq variables permettant de caractériser les variétés sont les rendements en sucre et en canne, la richesse saccharine, le taux de charbon et celui d'entre nœuds attaqués par E. saccharina. Ces variables quantitatives initiales corrélées ou non sont transformées en des variables non corrélées (composantes principales). Ce qui conduit au calcul d'une matrice diagonale de valeurs propres représentant les variances des individus sur les deux axes principaux qui participent en grande partie à la variance totale des individus. Ces axes permettent ainsi de mieux visualiser les individus les uns par rapport aux autres. 


\section{Résultats}

\subsection{Criblage variétal}

L'analyse de variance préalable au test de Newman-Keuls au seuil alpha $=5 \%$ montre un effet variétal hautement significatif $(p \leq 0,01)$ pour le rendement en sucre extractible, la tolérance au foreur de tiges et au charbon (tableau 1, 2 et 3). Le test de Newman-Keuls a donc permis de classer les variétés. Au bout de cinq ans, les variétés SP701143 et SP71-1406 surpassent le témoin par leurs rendements en sucre, avec 12,2 contre 11,08 tha${ }^{1}$ et 15,3 contre 14,03 t.ha $^{-1}$ (Tableau 1et 2). La première est plus tolérante au foreur et au charbon que NCo376 avec 1,21 \% d'ENA et 194,6 fouets.ha${ }^{1}$, contre $1,55 \%$ d'ENA et 11194,8 fouets. ha ${ }^{-1}$. La seconde (SP71-1406) est plus tolérante au charbon que le témoin NCo376 (455,4 contre 4
914,4 fouets. ha-1). Mais, elles possèdent des taux d'ENA équivalents (Tableau 3 ).

L'interaction variété ${ }^{*} a n n e ́ e ~ e s t ~ h a u t e m e n t$ significative $(p \leq 0,01)$ pour le TSE.ha ${ }^{-1}$. Les variations interannuelles influent sur le rendement en sucre des variétés. Le classement variétal change peu à partir de la troisième année pour les variétés commerciales. Aussi les conclusions de trois années d'essai sont-elles identiques à celles de cinq ans (durée de sélection en vigueur). Les variétés SP70-1143 et SP71-1406 auraient pu être sélectionnées en trois ans d'essai au lieu de cinq ans. Ce qui suggère l'intérêt de réduire la durée de sélection de deux ans (Tableau 1 et 2). Sur les cinq ans de sélection, aucune des cinq variétés présélectionnées n'a été retenue. Alors que, la moitié des variétés commerciales a été sélectionnée. Celles-ci présentent donc un taux d'adaptation plus élevé que les variétés présélectionnées.

Tableau 1 : Comparaison des moyennes de rendements en sucre extractible et effet des facteurs étudiés pour l'essai 1 conduit en début de saison de récolte sur cinq ans à Sucaf-Cl/Ferké 2 au Nord de la Côte d'Ivoire.

\begin{tabular}{|c|c|c|c|c|c|c|c|}
\hline \multirow{2}{*}{ Variétés } & \multicolumn{5}{|c|}{ TSE. ha $^{-1}$ des cultures de canne à sucre } & \multirow{2}{*}{$\begin{array}{l}\text { TSE.ha }{ }^{-1} \\
\text { pour } 3 \text { ans } \\
\text { d'essai }\end{array}$} & \multirow{2}{*}{$\begin{array}{l}\text { TSE.ha }^{-1} \\
\text { pour } 5 \text { ans } \\
\text { d'essai }\end{array}$} \\
\hline & Vierge (R0) & R1 & R2 & R3 & R4 & & \\
\hline B47258 & $9,35 \mathrm{ab}$ & $9,90 \mathrm{ab}$ & $9,40 \mathrm{a}$ & $9,14 \mathrm{a}$ & $8,27 \mathrm{a}$ & $9,55 b$ & $9,21 \mathrm{ab}$ \\
\hline B51129 & $9,02 \mathrm{ab}$ & 9,49 a & $9,81 \mathrm{a}$ & $9,37 \mathrm{a}$ & $9,81 \mathrm{a}$ & $9,44 b$ & $9,50 \mathrm{~b}$ \\
\hline FR80674 & $8,10 \mathrm{a}$ & $8,18 \mathrm{a}$ & $9,13 \mathrm{a}$ & $9,67 \mathrm{a}$ & 9,17 a & $8,47 \mathrm{a}$ & $8,85 \mathrm{a}$ \\
\hline FR80959 & $8,53 a b$ & $8,63 \mathrm{a}$ & 9,87 a & $8,71 \mathrm{a}$ & $8,57 \mathrm{a}$ & $9,01 \mathrm{ab}$ & $8,86 \mathrm{a}$ \\
\hline NCo376 & $9,47 a b$ & $10,19 a b$ & 11,26 b & $12,10 \mathrm{~b}$ & $12,37 b$ & $10,30 \mathrm{c}$ & $11,08 \mathrm{c}$ \\
\hline SP70-1143 & $10,10 \mathrm{~b}$ & $11,53 \mathrm{~b}$ & $12,85 \mathrm{~b}$ & $13,93 \mathrm{c}$ & $12,60 \mathrm{~b}$ & $11,49 \mathrm{~d}$ & $12,20 \mathrm{~d}$ \\
\hline Moyenne & 9,65 & 10,39 & 10,49 & 10,13 & 9,71 & 9,95 & 9,01 \\
\hline Ecart type Moyen & 1,19 & 1,41 & 2,07 & 1,90 & 1,06 & 1,38 & 0,71 \\
\hline Effet Variété & $0,04\left(^{*}\right)$ & $0,001\left(^{* *}\right)$ & $0,00\left({ }^{* \star}\right)$ & $0,00\left({ }^{* *}\right)$ & $0,00\left({ }^{* \star}\right)$ & $0,00\left({ }^{* *}\right)$ & $0,00\left({ }^{* *}\right)$ \\
\hline Effet Année & - & - & - & - & - & $0,00\left(^{* \star}\right)$ & $0,00\left(^{* *}\right)$ \\
\hline Effet Bloc & 0,53 & 0,99 & 0,79 & 0,24 & 0,16 & 0,60 & 0,10 \\
\hline Inter Var*An & - & - & - & - & - & 0,10 & $0,00\left(^{\star \star}\right)$ \\
\hline
\end{tabular}

NB

- Ri : repousse (i) correspondant à l'année (i) de sélection;

- les moyennes suivies des mêmes lettres (unique ou double) dans la même colonne ne sont pas significativement différentes au seuil de $5 \%$ selon le test de Newman-Keuls ;

- effet significatif $\left({ }^{*}\right)$; effet hautement significatif $\left({ }^{* *}\right)$. 
Tableau 2 : Comparaison des moyennes de rendements en sucre extractible et effet des facteurs étudiés pour l'essai 2 conduit en début de saison de récolte sur cinq ans à Sucaf-Cl/Ferké 2 au Nord de la Côte d'Ivoire.

\begin{tabular}{|c|c|c|c|c|c|c|c|}
\hline \multirow{2}{*}{ Variétés } & \multicolumn{5}{|c|}{ TSE.ha $^{-1}$ des cultures de canne à sucre } & \multirow{2}{*}{$\begin{array}{c}\text { TSE.ha' } \\
\text { pour } 3 \text { ans } \\
\text { d'essai }\end{array}$} & \multirow{2}{*}{$\begin{array}{l}\text { TSE.ha' } \\
\text { pour } 5 \text { ans } \\
\text { d'essai }\end{array}$} \\
\hline & Vierge (R0) & R1 & $\mathbf{R 2}$ & R3 & R4 & & \\
\hline Co449 & $11,31 \mathrm{~b}$ & $12,78 \mathrm{~b}$ & $13,97 \mathrm{bc}$ & $13,34 a b$ & $12,43 a b$ & $12,69 \mathrm{c}$ & $12,77 \mathrm{~b}$ \\
\hline FR81254 & $9,21 \mathrm{a}$ & $12,78 \mathrm{~b}$ & $12,43 \mathrm{a}$ & $13,10 a b$ & $12,06 \mathrm{ab}$ & $11,47 \mathrm{~b}$ & $11,92 a b$ \\
\hline FR81258 & $10,77 \mathrm{~b}$ & $12,69 \mathrm{~b}$ & $13,45 a b$ & $13,03 a b$ & 10,99 a & $12,30 \mathrm{c}$ & $12,19 a b$ \\
\hline FR8183 & $8,94 \mathrm{a}$ & $10,54 \mathrm{a}$ & $11,87 \mathrm{a}$ & 12,26 a & $13,19 a b$ & $10,45 \mathrm{a}$ & 11,36 a \\
\hline NCo376 & $11,41 \mathrm{~b}$ & $13,80 \mathrm{bc}$ & $15,56 \mathrm{bc}$ & $15,28 \mathrm{bc}$ & $14,08 \mathrm{~b}$ & $13,59 d$ & $14,03 \mathrm{c}$ \\
\hline SP71-1406 & $12,35 \mathrm{c}$ & $14,85 \mathrm{c}$ & $15,69 \mathrm{c}$ & $17,60 \mathrm{c}$ & $16,03 \mathrm{c}$ & $14,30 \mathrm{~d}$ & $15,31 d$ \\
\hline Moyenne & 10,67 & 12,91 & 13,83 & 14,10 & 13,13 & 12,47 & 12,93 \\
\hline Ecart type Moyen & 1,34 & 1,43 & 1,58 & 1,99 & 1,76 & 1,40 & 1,48 \\
\hline Effet Variété & $0,00\left(^{* \star}\right)$ & $0,00\left(^{* *}\right)$ & $0,00\left(^{* *}\right)$ & $0,00\left(^{\star *}\right)$ & $0,00\left({ }^{* \star}\right)$ & $0,00\left(^{* *}\right)$ & $0,00\left(^{* *}\right)$ \\
\hline Effet Année & - & - & - & - & - & $0,00\left(^{* *}\right)$ & $0,00\left(^{* \star}\right)$ \\
\hline Effet Bloc & 0,18 & 0,10 & 0,72 & 0,58 & 0,11 & 0,14 & $0,00\left(^{* \star}\right)$ \\
\hline Inter Var*An & - & - & - & - & - & 0,37 & $0,03\left(^{*}\right)$ \\
\hline
\end{tabular}

NB :

- Ri : repousse (i) correspondant à l'année (i) de sélection;

- les moyennes suivies des mêmes lettres (unique ou double) dans la même colonne ne sont pas significativement différentes au seuil de $5 \%$ selon le test de Newman-Keuls ;

- effet significatif $\left(^{*}\right)$; effet hautement significatif $\left(^{* *}\right)$.

Tableau 3 : Taux de fouets charbonneux et d'entre-nœuds attaqués pour les essais 1 et 2 conduits en début de saison de récolte sur cinq ans à Sucaf-Cl/Ferké 2 au Nord de la Côte d'Ivoire.

\begin{tabular}{|c|c|c|c|c|c|}
\hline \multicolumn{3}{|c|}{ Essai 1} & \multicolumn{3}{|c|}{ Essai 2} \\
\hline Variétés & $\begin{array}{c}\text { Charbon } \\
\text { (fouets.ha }^{-1} \text { ) }\end{array}$ & ENA\% & Variétés & $\begin{array}{c}\text { Charbon } \\
\left(\text { fouets.ha }^{-1}\right)\end{array}$ & ENA\% \\
\hline B47258 & $19171,80 \mathrm{c}$ & $0,97 \mathrm{~b}$ & Co449 & $22,80 \mathrm{a}$ & $0,92 \mathrm{a}$ \\
\hline B51129 & $473,00 a$ & $1,19 \mathrm{c}$ & FR81254 & $77,60 \mathrm{a}$ & $1,74 \mathrm{~b}$ \\
\hline FR80674 & $6025,60 \mathrm{ab}$ & $1,51 \mathrm{~d}$ & FR81258 & $58,60 \mathrm{a}$ & $0,90 \mathrm{a}$ \\
\hline FR80959 & 538,80 a & $0,69 \mathrm{a}$ & FR8183 & $346,00 \mathrm{a}$ & $1,20 \mathrm{a}$ \\
\hline NCo376 & $11194,80 \mathrm{~b}$ & $1,55 \mathrm{~d}$ & NCo376 & 4914,40 b & $1,26 a$ \\
\hline SP70-1143 & 194,60 a & $1,21 \mathrm{c}$ & SP71-1406 & $455,40 a$ & $0,94 \mathrm{a}$ \\
\hline Moyenne & 6266,40 & 1,19 & & 979,1 & 1,16 \\
\hline Ecart type Moyen & 5378,30 & 0,39 & & 2164,5 & 0,29 \\
\hline Effet variété & $\left.0,00{ }^{(* *}\right)$ & $0,00\left(^{* \star}\right)$ & & $0,01\left(^{*}\right)$ & $0,00\left(^{* * \star}\right)$ \\
\hline
\end{tabular}

NB :

- Ri : repousse (i) correspondant à l'année (i) de sélection;

- les moyennes suivies des mêmes lettres (unique ou double) dans la même colonne ne sont pas significativement différentes au seuil de $5 \%$ selon le test de Newman-Keuls ;

- effet significatif $\left(^{*}\right)$; effet hautement significatif $\left({ }^{* *}\right)$. 


\subsection{Caractérisation des groupes de variétés}

Les paramètres de richesse saccharine et les rendements en canne et en sucre sont corrélés positivement au facteur 1 (axe 1) qui participe à 54 $\%$ de la variance totale des variétés dans le premier essai, avec respectivement $r=0,77 ; 0,95$ et 0,99 . Alors que les paramètres phytosanitaires que sont les taux de charbon et d'entre nœuds attaqués sont corrélés positivement au facteur 2 (axe 2) qui participe à $27 \%$ de la variance totale de l'essai, avec $r=0,66$ et 0,78 . C'est à partir de ces corrélations entre les variables et les facteurs (1 et 2) que la dispersion (positionnement) des variétés dans le plan factoriel ou plan de dispersion (figure 1) est interprétée.

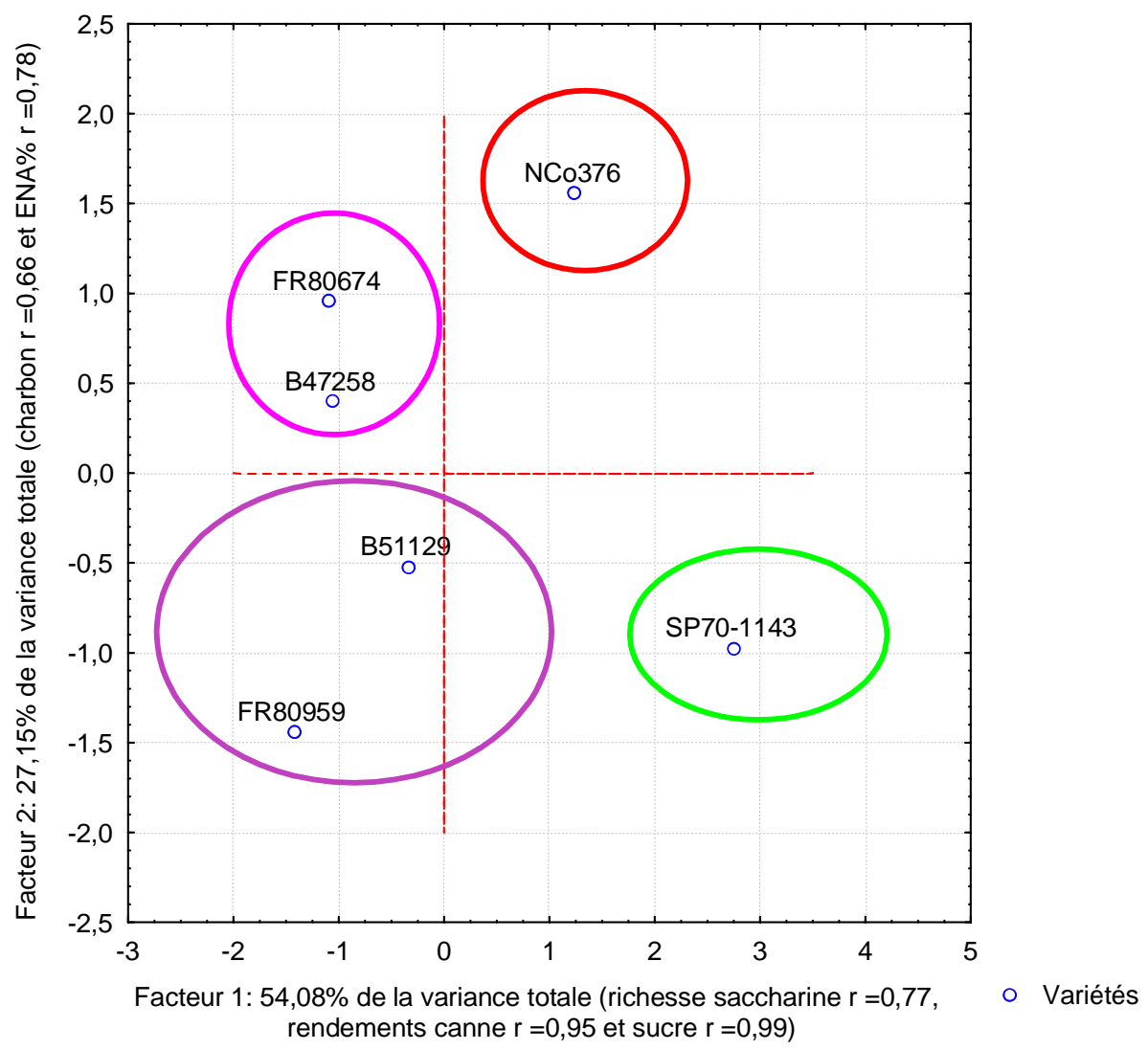

Figure 1 : Groupes de variétés déterminés par l'analyse en composantes principales relative à l'essai 1 conduit en début de saison de récolte sur cinq ans à Sucaf-Cl/Ferké 2, au Nord de la Côte d'Ivoire.

Ainsi, selon la figure 1, quatre groupes de variétés se distinguent:

- le premier groupe : témoin NCo376 qui est caractérisée par des qualités agrotechnologiques moyennement bonnes et une forte sensibilité au foreur de tiges et au charbon ;

- le second groupe : SP70-1143 qui est très peu sensible au foreur de tige et au charbon. Une richesse en saccharose, des rendements en canne et en sucre élevés caractérisent en outre cette variété.

- les variétés FR80959 et B51129 constituent le troisième groupe. Elles sont caractérisées par des qualités agrotechnologiques médiocres, mais sont très résistantes au foreur de tiges et au charbon: 
- le dernier groupe comprend FR80674 et B47258 qui sont sensibles au foreur de tiges et au charbon, et présentent des qualités agro-technologiques médiocres.

Concernant l'essai 2, I'ACP montre que le facteur 1 (axe 1 de la figure 2) participe à $43 \%$ de la variance totale de l'essai et le facteur 2 (axe 2 de la figure 2) contribue à $25 \%$ de cette variabilité. II apparait que la richesse saccharine d'une part et les rendements en canne et sucre d'autre part, sont corrélés positivement au facteur 1 , avec respectivement $r=0,39 ; 0,95$ et 0,87 . Quant aux paramètres phytosanitaires de tolérance au charbon et au foreur, ils sont corrélés négativement au facteur $2(r=-0,95$ et $-0,39)$. Le paramètre de tolérance au foreur de tiges est aussi corrélé négativement au facteur $1(r=-0,54)$. Le plan de dispersion des individus de l'ACP pour cet essai fait donc ressortir trois groupes de variétés (figure 2) :

- la variété témoin NCo376 constitue le premier groupe. Elle est caractérisée par des qualités agro-technologiques moyennement bonnes, mais est très sensible au charbon et au foreur de tiges ;

- les variétés Co449, FR81258, FR81254 et FR8183 (groupe 2) sont très résistantes au foreur de tiges et au charbon, avec des rendements et des qualités technologiques médiocres ;

- la variété SP71-1406 (groupe 3) est caractérisée par les meilleures performances de rendement agronomique et de qualités technologiques. Du point de vue phytosanitaire, elle s'avère tolérante au foreur de tiges et au charbon.

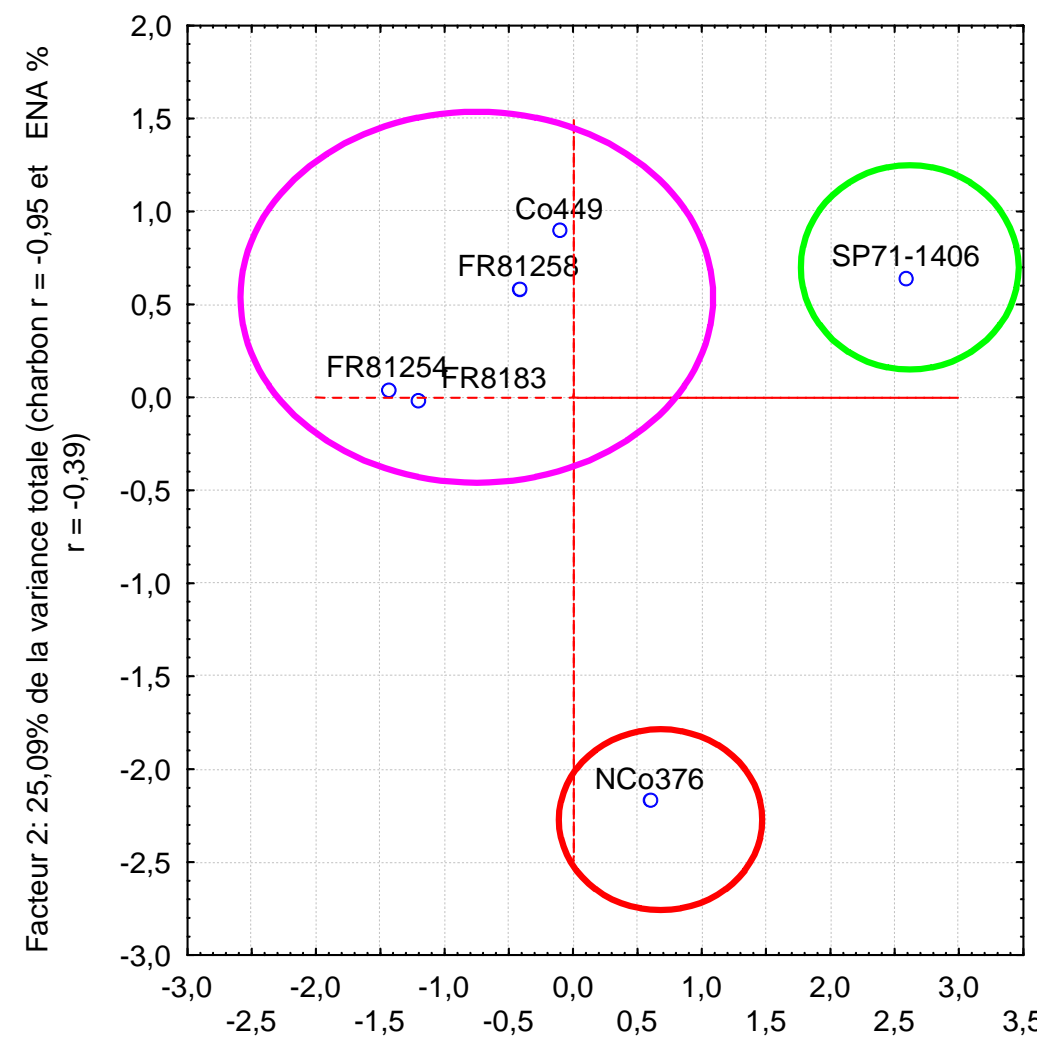

Facteur 1: 43,13\% de la variance totale (richesse saccharine $r \quad \circ$ variétés $=0,39$, rendements canne $r=0,95$ et sucre $\quad r=0,87$ )

Figure 2 : Groupes de variétés déterminés par l'analyse en composantes principales relatives à l'essai 2 conduit en début de saison de récolte sur cinq ans à Sucaf-Cl/Ferké 2, au Nord de la Côte d'Ivoire. 


\section{Discussion}

Les résultats des essais ont montré que deux variétés sont prometteuses. SP70-1143 est une variété à fort rendement en canne et en sucre (102 t canne.ha ${ }^{-1}$ et 12 t sucre.ha- $\left.{ }^{-1}\right)$. Les tests préindustriels de cette variété ont été concluants et elle a été cultivée en 2008 sur $15 \%$ (774,3 ha) de la sole cannière du site. La variété SP71-1406 quant à elle, révèle une très bonne richesse saccharine, avec des rendements en canne et en sucre élevés (128 t canne.ha-1 et $15 \mathrm{t}$ sucre.ha- ${ }^{-1}$ ). C'est une variété auto-épaillante, à port érigé, se prêtant à la coupe mécanique en vert et à la culture en rangs jumelés. Ces variétés se sont avérées très résistantes vis-à-vis des bio-agresseurs majeurs de la canne à sucre à Ferké 2 que sont $E$. saccharina et $U$. scitaminea, comparativement au meilleur témoin NCo376. Selon Mario et al. (1995), Pereira (2001) et Matsuoka et al. (2009), ces variétés appartiennent au même groupe génétique (ancêtres communs, adaptation aux sols pauvres et aux mêmes types d'environnements). Elles ont permis au Brésil de se hisser parmi les premiers producteurs de sucre de 1980 à 1995. Aujourd'hui, elles sont utilisées au Brésil comme parents et témoins dans les programmes de sélection. Ainsi, elles pourraient remplacer à terme les variétés NCo376 et Co957 qui sont sensibles à la maladie du charbon en Côte d'Ivoire. Ces dernières représentaient, $15 \%$ et $30 \%$ de la répartition variétale à Ferké 2 , en 2008. Sachant que le poids moyen d'une canne de NCo376 est de 0,5 à $0,7 \mathrm{~kg}$, les pertes de productions occasionnées par le charbon sur cette variété sont de 1932,5 à 6493,2 t en 2008. La situation reste préoccupante en culture industrielle où, l'infestation de NCo376 par le charbon a atteint 4599 fouets charbonneux. ha ${ }^{-1}$, soit 3,22 t.ha-1 correspondant à $2500 \mathrm{t}$ de perte en 2008 . Les pertes dues au charbon varient ainsi de 20 à 30 $\%$ du rendement final (Frison \& Putter, 1993).

Les essais se sont soldés par la sélection de 50 $\%$ des variétés commerciales contre un taux nul de variétés présélectionnées. Les premières seraient donc mieux adaptées aux conditions agro-pédologiques de Ferké 2 que les dernières ; confirmant ainsi les résultats de Péné \& Tuo (2007) sur le même site. Les variétés présélectionnées ont constitué pendant plus de deux décennies une part importante du matériel végétal importé en Côte d'Ivoire. Elles étaient présumées pouvoir s'adapter à divers environnements, la pression de sélection étant restée faible quant au paramètre environnemental. Cependant, comme en Côte d'Ivoire, ces variétés s'adaptent plus ou moins difficilement dans d'autres pays où quelques unes n'ont atteint le stade préindustriel qu'à des dates récentes (Oriol \& Domaingue, 2008). Ces variétés étaient en cours de développement et leur comportement industriel était peu connu. Les introductions futures de variétés de canne en Côte d'Ivoire devraient donc donner la priorité aux variétés commerciales. Parmi celles acquises récemment par la SUCAF, les origines mauriciennes sont issues de schéma privilégiant l'élitisme (Ramdoyal \& Autrey, 2005). De tels génotypes offrent de bonnes perspectives de productivité pour la filière canne à sucre ; car ils pourraient révéler un niveau de performance plus élevé (Casadebaig, 2008, Matsuoka et al., 2009). En effet, il a été prouvé en Australie et en Floride que les nouvelles variétés prometteuses contribuaient entre 69 et $75 \%$ du gain de productivité en sucre par an (Domaingue et al., 2008). La durée du schéma de sélection de la canne à sucre en vigueur était de 11 à 15 ans, avec trois étapes (Marion et al.,1998 ; Péné \& Déa 2000). La première étape était réalisée en trois ans, à la station du CNRA/Ferké au profit des complexes du Nord (Ferké 1, Ferké 2, Borotou). La deuxième étape (concernant cette étude), conduite dans les complexes sucriers durait cinq ans et la troisième consistait en des tests d'observations portant sur les variétés prometteuses en trois ans. Les résultats des essais, dans les conditions de Ferké 2 , montrent que les variétés réagissaient différemment selon les années. Cependant leur classement reste le même à partir de la troisième année ; la sélection des variétés commerciales pouvant être menée en trois ans au lieu de cinq ans pour la deuxième étape de sélection. Aussi, la première étape de criblage dont le rôle était de réduire le nombre de variétés à introduire sur les complexes sucriers s'avère-t-elle superflue et peut être supprimée. En définitive, l'étape expérimentale dans ce schéma pourrait se faire en une seule étape de trois ans au lieu de deux étapes en trois et cinq ans. En outre, les trois années de tests d'observations sont insuffisantes pour apprécier la tenue en repousse d'une variété, puisqu'à Ferké 2 , le cycle moyen de culture dure six ans. Cette question ne saurait donc être traitée 
définitivement par l'expérimentation seule, au risque que la durée de sélection soit trop longue. C'est pourquoi, après les trois années de test d'observation en conditions commerciales sur 100 ha, chaque nouvelle variété devra continuer à être observée au fil des repousses jusqu'à la replantation. Toutefois, après les trois premières années de tests, les variétés performantes aussi bien en plantation qu'à l'usine doivent être adoptées commercialement et cultivées sur des superficies bien plus importantes que les 100 ha requis par les tests d'observations. La faiblesse de la stratégie expérimentale présentée ici réside dans le fait que l'étude n'ait pas pris en compte dès le départ les principaux types de sol existants sur le site. Il aurait été préférable de répéter chaque essai au moins dans les deux principaux types de sol (ferrallique et hydromorphe). Ce qui aurait permis un zonage dans le développement des variétés comme à La Réunion et à Maurice (Ramdoyal \& Autrey, 2005 ; Barau, 2008).

\section{Conclusion}

Les variétés SP70-1143 et SP71-1406, d'origine brésilienne, se sont avérées prometteuses parmi celles évaluées en deux essais conduits chacun sur cinq ans à SUCAF-Ferké 2. Elles peuvent remplacer les variétés obsolètes devenues trop sensibles aux ennemis majeurs de la canne à sucre sur les complexes de SUCAF/Ferké. En outre, il apparait qu'au bout de trois ans, la durée de la sélection n'influence pas le classement des variétés commerciales selon leurs performances en termes de sucre extractible et de sensibilité aux stress biotiques endémiques. Ce qui suggère l'intérêt de privilégier l'introduction des variétés commerciales et de mettre en œuvre une seule étape de sélection sur trois ans. Suivront enfin les tests préindustriels sur également trois ans. Si la phase expérimentale du schéma de sélection de la canne à sucre en Côte d'Ivoire est ramenée à une seule étape de trois ans, celui-ci durera 6 ans au lieu de 11 ans.

\section{Références citées}

Barau L., 2008. Les premiers résultats de la sélection multilocale du CERF. In: 4 ème Rencontre Internationale de I'AFCAS, Guadeloupe. pp. 1-6.
Bigot S., Yao T.B., Oszwald J., \& Diedhiou A., 2005. Facteur de variabilité pluviométrique en Côte d'Ivoire et relations avec certaines modifications environnementales. Sécheresse 16 (1). 5-13.

Casadebaig M.P., 2008. Analyse et modélisation des interactions génotype-environnementconduite de culture : application au tournesol (Helianthus annuus). Thèse de l'institut national polytechnique de Toulouse. 196 pp.

D'Hont A., Grivet L., Feldmann P., Rao S., Berding N., \& Glaszmann J.-C. 1996. Characterization of the double genome structure of modern sugarcane cultivars (Saccharum spp.) by molecular cytogenetics. Mol. and Gen. Genetic. 250: 405-413.

Domaingue R., Hoarau J.Y., Oriol P. \& Roques D., 2008. Le progrès génétique chez la canne à sucre : bilan, enseignement et perspectives. In: $4^{\text {ème }}$ Rencontre Internationale de l'AFCAS. Guadeloupe. pp. 1-24.

Eldin M., 1971. Le climat. In: Le milieu naturel de la Côte d'Ivoire. Mémoire ORSTORM, N 50. Paris 391. pp. 73-108.

Feldmann P., Oriol P., Daugrois J.H., Boisne-Noc R., Gelabale G., Gravillon M.C., Joseph S., Navis P., Ragouton M., Sapotille J. \& Toubi L., 1998. Amélioration variétale en Guadeloupe. In: Canne à sucre. Cirad, Département des cultures annuelles: pp.20-22.

Frison E.A. \& Putter C.A.J., 1993. Technical guidelines for the safe movement of sugarcane germplasm. Food and Agriculture Organization of the United Nations/ International Board for Plant Genetic Resources (FAO/IBPGR) Rome, Italy http:// www.sgrp.cgiar.org/publications/field/ invitroguidelines 06/09/2006.

Goebel F-R., Way, M.J, \& Conlong, D., 2008. La gestion des foreurs de la canne à sucre, l'environnement et les pratiques culturales: synthèse des résultats et perspectives. In: 4 ème Rencontre Internationale de I'AFCAS. Guadeloupe. pp. 13-24.

Goebel F-R., Way, M.J, \& Gossard, C., 2005. The status of Eldana saccharina (Lepidoptera: Pyralidea) in South Africa sugar industry based on regular survey data. Proceeding of the South Africa Sugarcane Technologists Association, 79: 337-346. 
Hoarau M., 1970. Utilisation de la presse hydraulique pour la détermination de la richesse saccharine de la canne à sucre. In : La canne à sucre, par Fauconnier et Bassereau. IRAT, Maisonneuve et Larose. pp. 387-419.

Hoareau S., Hoareau W., Petit A. \& Corcodel L., 2008. Etat des lieux de la polarisation proche infrarouge sur les différents produits de l'industrie sucrière réunionnaise. In : $4^{\text {ème }}$ Rencontre Internationale de I'AFCAS. Guadeloupe. pp. 1-15.

John B.H., 1975. Reconnaissance des sols dans la zone du projet de Ferké 2. Rapport des Services de l'aménagement des ressources forestières, Université d'Ibadan, Nigéria. 71 pp.

Mario P.C., Raphaël A., Marcelo D.A.S., Celso V.P., Antonio P.D.C., Marcos G.D.A.L., Léo Z., Maria B.S. \& Pery F., 1995. Sugarcane breeding: Evaluation of clones obtained by hybridizations in 1980 and selected in Jau Region, State of Sao Paulo, Brazil. Bragantia 54 (1): 95-102.

Marion D., Bamba M., Tuo K., Keï A. \& Eboi P., 1998. Sélection variétale en Côte d'Ivoire. In: Canne à sucre. Cirad, Département des cultures annuelles. pp. 25-26.

Matsuoka S., Ferro J., \& Arruda P., 2009. The Brazilian experience of sugarcane ethanol industry. In Vitro Cell. Dev. Biol.-Plant 45: 372-381.

Oriol P. \& Domaingue R., 2008. Sélection variétale de la canne à sucre : un levier pour soutenir la compétitivité des exploitations agricoles du Sud. In : Canne à sucre. Cirad, Montpellier. pp. 1-2. http://www.cirad.fr 22/05/2008.

Paulet F., \& Glaszmann J.-C., 1994. Les biotechnologies en soutien à la diffusion variétale chez la canne à sucre. Agric. et dév. 2: 55-61.
Péné C.B. \& Tuo K., 2007. Early and late-season screening of sugarcane varieties in northern Ivory Coast. In: Actes du 26ème congrès ISSCT, Durban. pp. 110.

Péné C.B., \& Déa G.B., 2000. Amélioration variétale de la canne à sucre en Côte d'Ivoire. $\ln : 1^{\mathrm{er}}$ atelier régional sur l'amélioration variétale de la canne à sucre en Afrique de l'Ouest et Centrale. Yamoussoukro. pp. 98-102.

Péné C.B., Chopart J.L. \& Assa A., 1997. Gestion de l'irrigation à la parcelle en culture de canne à sucre (Saccharum officinarum L.) sous climat tropical humide, à travers le cas des régions nord et centre de la Côte d'Ivoire. Sécheresse 8 (2). 87-98.

Pereira B.M.H., 2001. Study of genetic divergence in sugarcane varieties grown in Brazil using the parentage coefficient. Inter. Sug. journ. 103 (1231). 294-295.

Ramdoyal K. \& Autrey L.J.C., 2005. Amélioration de la canne à sucre-stratégies, performances des variétés commerciales en 2004 et homologation d'une nouvelle variété. Rev. Agric. et Sucr. de lîle Maurice 84 (1, 2 \& 3). 3-25.

Sreenivasan T.V., Ahloowalia B.S., \& Heinz D.J., 1987. Cytogenetics. In: Sugarcane improvement through breeding. DJ Heing, ed. Elsevier, Amsterdam 11: 211-253.

Tran M., 1981. Reconnaissance des principaux foreurs de tiges du riz, du maïs et de la canne à sucre en Côte d'lvoire. Initiationsdocumentations techniques, $\mathrm{N}^{\circ} \mathbf{4 8}$. ORSTOMParis : $23 p$.

Wada A. C., 2003. Control of sugarcane smut disease in Nigeria with fungicides. Crop protect. 22: 45-49.

Yoseph A., Van den Berg J., \& Colong D.E., 2008. Farmers' perceptions of sugarcane stem borers and farm management practices in the Amhara region of Ethiopia. Inter. Journ. of Pest Manag. 54 (3): 219-226. 
